On the Chromatic Aberration of the Eye in relation to the Perception of Distance

This content has been downloaded from IOPscience. Please scroll down to see the full text. 1875 Proc. Phys. Soc. London 2157

(http://iopscience.iop.org/1478-7814/2/1/315)

View the table of contents for this issue, or go to the journal homepage for more

Download details:

IP Address: 149.171.67.164

This content was downloaded on 10/09/2015 at 19:07

Please note that terms and conditions apply. 
May $12,1877$.

XV. On the Chromatic Aberration of the Eye in relation to the Perception of Distance. By Silvanus P. Thompson, B.A., B.Sc.

1. The experimental researches of Wollaston, Arago, Fraunhofer, Young, Matthiesen, Brewster, Müller, and Helmholtz have left no doubt that the eye has the defect of chromatic aberration. Rays of light of dissimilar colour, starting in the same direction from any luminous point and entering the eye, do not all come to focus upon the retina at once, but the foci for differently coloured rays are differently situated with respect to the retina.

Hence, so far as the perception of the distance of a luminous object is dependent upon the accurate focusing of rays upon the retina of the eye, so far the want of adjustment in the eye introduces a source of errör into the data of our perception. If the eye be not achromatic, a blue object and a red olject of equal dimensions placed side by side cannot be in focus at once, and lence must appear either unequally distant or else of unequal magnitude.

2. The object of this paper is to demonstrate that the fact thus briefly sketched ont affords a true and legitimate explanation of certain empirical rules of artistic practice relative to the pictorial expression of distance. The existence and universal recognition of such empirical rules has presented itself as a problem to the mind of the writer for the past six years; but it is only within the last few months that the solution now offered has been adopted by him.

It is proposed therefore :-

I. To enumerate the various data dependent on the eye and not upon the feet or other limbs, for the formation of a perception of distance.

II. To discuss the weight to be attached to the several data under various circumstances.

III. To inquire how far these data may be dependent upon the apparent colour of an ohject, or upon the formation of an exact focus upon the retina of the eye. 
1V. To give a brief account of some experimental results, together with conclusions derived therefrom.

\section{Data for the Perception of Distanre.}

3. The following nine data may be enumerated as contributing to the formation of a pereeption of distance.

$4(a)$. Apparent motion of oljects caused by motion of the observer. If we move from one place to another, or alter the position of the hear, the risible contiguration of objects suffers a corresponding change, which by association with previous experience suggests an external system, of whose distance this "parallax" affords us an idea.

5 (b). Apparent change of configuration of objects according as they are riewed with one eye or the other. This affords " "parallax" similarly to $(a)$, though on a more limited scale.

if (c). The muscular sensation of convergence or divergence of the two eyes when directed towards an object. This is a genuine muscular perception, though guided by the optical perception of want of positional adjustment when the corresponding parts of the two images do not fall upon the corresponding points of the two retinx.

$7(d)$. Retinal magnitude of an object as appreciated by the muscular effort of turning the eyeball so as to bring the parts of the image successively into the centre of the field of vision.

8 (e). Retinal magnitude as a sensation of excitement of a certain area of nerve matter of the retina.

$9(f)$. The muscular sensation of effort of the ciliary muscle in the adjustnent of the eye to exact focus. The perception of exactness or inexactness of focus which guides the muscular action is, too, an optical effect in itself of little value as a means of estimating distance, cxcept in so far as it controls the required muscular contraction.

$10(y)$. Binocular dissimilarity of the images on the two retinæ, which, conspiring to give one mental impression, present slight differences that suggest the idea of distance.

$11(h)$. Linear perspective. Under this ter'm I propose to include, beside the usually understood play of lines and diminishing apparent magnitudrs commonly spoken of as 
perspective, such artificial means of estimating distance as are afforded by comparison with objects of known sizo (figures, cattle, \&c.), and such notions of position, surface, \&c. as can be drawn from comparison with houses or other objects of known form and magnitude.

12. (i) Aerial Perspective, in its widest sense. On this point Helmboltz has most aptly remarked* that the brain has the same means for the appreciation of relative distance as are employed by the painter on his canvas ; and he enumerates amongst these means strong shadows, bold relief in the foreground, obscurity of atmosphere, and dimness of outline, in addition to contrasts with objects of known size or form. The term, however, is often restricted to the peculiar alteration of the colour of objects due to the intervening atmosphere $\dagger$.

\section{Relative Importance of foregoing Data.}

13. It is obvious that the considerations advanced in (a), $(b)$, and (c) are foreign to the present inquiry, though of enormous importance in the estimation of distances in general; for there can be no doubt that where these means are available, the mind is guided to a large extent by them in the formation of an estimate of distance, unless carefully trained to disregard them and to depend on other circumstances, or unless, as may happen to individuals, some physical injury has occurred to render the eye abnormal. With respect to the consideration advanced in (c), I have some reason to doubt whether we really do judge apparent distance by the muscular sensations of the external and internal recti muscles of the eye. Stereoscopic pictures appear to me equally " solid" whether in a Wheatstone's reflecting stereoscope, a Brewster's lenticular with prism-shaped lenses, or a Helmholtz's with parallel axes, or by superimposition by effort of the recti muscles, in each of which cases a different convergence is

* 'Popular Lectures,' English edition, p. 281.

$\dagger$ Mr. Ruskin's definition of aerial perspective is, from an artistic point of view, of value. "Aerial perspective is the expression of space by any means whatsoever, sharpness of edge, vividness of colour, \&c. assisted by greater pitch of shadow, and requires only that objects should be detached from each other by degrees of intensity in proportion to their distance."'Modern Painters,' vol. i. p. 139. 
required. Moreover it would be very inconvenient if our estimate of distance depended on the action of these muscles, as they are peculiarly liable to fatigue.

14. Retinal magnitude, to use the term adopted by Wheatstone, whether regarded in its muscular $(d)$ or its optical (e) sense, affords only an indirect means of estimating distance by association with ideas previously acquired concerning the retinal dimensions of objects of known size at known distances. Nothing is more easy than deception arising from this cause. We constantly hear the magnification of the telescope spoken of as though the enlargement of the retinal image produced the same effect as if the object were brought so near to the eye as to yield a retinal image of equal area. This is evidently not the case, as the distant object, however much magnified in the telescopic image, is still seen by rays travelling in paths nearly parallel to each other, and not containing angles as great as those contained by rays proceeding from an object really near. Hence a telescopic presentation of an object is flatter than the object itself appears when brought sufficiently near to give a retinal image of equal magnitude.

15. The fallacy of judging distances by apparent magnitude is made evident by the absurd comparisons often made between the sun or moon and other objects, some individuals comparing them to the size of a coin, a plate, or a cart-wheel. Comparatively few persons could at once tell you correctly which appears the larger, the setting sun or a threepennypiece held at arm's length-the fact being that the angular magnitude of the latter exceeds that of the former.

16. The following experiment * illustrates a remarkable power of accommodation of the eye to the requirements of the mind in respect of retinal magnitude. Place under a stereoscope a penny and a halfpenny, having the "heads" of both coins placed upwards and similarly. You shall see but one coin when looking with the two eyes, especially if you expect no dissimilarity and do not use the eyes alternately. There are, on the other hand, experiments of Helmholtz, Fechner,

- My attention was first drawn to this curious fact by Mr. Joseph Beck, of the eminent firm of Smith and Beck. 
and Volkmann * which go to prove that the retinal magnitude, as appreciated by the muscular sweep of the eye, affords a fairly accurate means of comparison of the angular magnitudes and positions of two objects in the field of vision whose images can be successively brought upon the same spot of the retina. This involves, however, the question of the persistence of visual impressions, without which such a comparison of retinal magnitudes might be quite fallacious.

17. The sensation $(f)$ of tension of the ciliary muscle is a very delicate means of estimating near distances, and is a sensation capable of training to a considerable nicety. In October 1870 the writer was unable to perceive any difference of strain of adjustment to vision between distances of 4 yards and 400 yards. At the present time he is conscious of a difference of tension when the eye is directed to objects 15 yards and 300 yards distant respectively, and this with either eye. $I$ insist on this means of estimation of distance, because it is, for monocular vision, of even more importance than the muscular sensation of convergence of the optic axes is for binocular vision.

18. The researches of Wheatstone, Brewster, Dove, Helmholtz, Brücke, Müller, Rood, Volkmann, and Wundt upon the stereoscopic presentation of objects resulting from the binocular dissimilarity of the two retinal images $(g)$ illustrate the importance of this fact of binocular vision. Wheatstone's pseudoscope proved the point by a reductio ad absurdum; and an equally conclusive proof is obtained by cutting a stereoscopic slide in two and placing the halves in laterally reversed position under the stereoscope. I have, however, experimentally found that considerations of colour may sometimes outweigh the judgment founded by the eye upon the element of dissimilarity. I drew a pattern of two squares of four blue lines each upon paper, and within each, and outside each, centrally described circles in red. When this pattern was viewed in the stereoscope, the blue squares seemed to draw back from the eye. I then traced some of Möser's crystal forms from the well-known lithographed stereoscopic slides, colouring the front lines of the crystals with ultramarine, and the back lines

* Helmholtz, 'Popular Lectures,' p. 279; and Physiologische Optik, pp. 522-524 and 542. 
with vermilion. Several, though not all, of these when placed in the stereoscope refused to appear of their true form, the blue lines, whose positions would stereographically give a front presentment, retiring behind the red lines of the crystal.

That the mind does, however, habitually make use of the dissimilarity of binocular vision is evident from the old trick of looking with one eye through a short tube while attempting to walk up to a suspended ribbon and cut it with a pair of scissors. Yet persons who have the use of but one eye find no difficulty in performing the task; and, with practice, the feat becomes easy if the distance be carefully estimated by the sensations of ocular focus. The linear distance between the two eyes, however, sets a limit to the range of habitual estimates of distance founded on the fact of binocular dissimilarity ; and, as Mr. Ruskin has observed *, it is possible to see, and to see in focus together, the extreme distance and the middle distance of a landscape, though the foreground and distance cannot so be seen together.

19. I cannot here dwell upon questions involving linear perspective $(h)$. The various considerations upon which linear perspective becomes a basis for the perception of distance are purely associative-mental rather than optical, geometrical rather than physical.

20. I pass on, therefore, to aerial perspective (i); the various definitions of which may be summed up in one as the expression of distance by colour. A distant hill looks bluer than a less distant one by reason of the slight opacity of the intervening atmosphere ("sky"); and its blueness is in some manner, to a normal eye, a far truer measure of its distance than any one of the considerations yet treated of. The blueness thus existing in nature varies with the changing conditions of atmosphere, and hence is liable to be fallacious when the atmo-

- Mr. Ruskin advances this point in defence of the practice of landscape-painters in leaving foregrounds sketchy and unfinished, maintaining that they should exhibit "a decisive imperfection, a firm but partial assertion of form which the eye feels indeed to be close home to it, and yet cannot rest upon, nor cling to, nor entirely understand, and from which it is driven away of necessity, to those parts of distance on which it is intended to repose."- Modern Painters,' vol. i. pp. 183 and 184, note. Mr. Ruskin adds that Turner was the first to introduce this treatment into lindscape art. 
spheric conditions are unfamiliar. Thus in Smith's 'Optics' it is narrated of Bishop Berkeley that, when travelling in Italy and Sicily, he thought the cities as he approached them two or three miles too near, by reason of the unfamiliar transparency of the air.

\section{Bearing of Chromatic Aberration upon the foregoing Data.}

21. Taking the chromatic aberration of the eye as an established matter *, it is now time to inquire how far these various considerations are affected by the fact that rays of different colours have different focal length in the eye.

22. Obviously all estimates of distance which depend on retinal magnitude are liable to be influenced by colour. This may easily be verified by taking two equal disks of red and blue paper and placing them upon a black background. It will be found impossible to get a perfectly distinct image of both at once, one or other being out of focus, and therefore blurred at the edge and larger; or if no difference in size appears, one (tho blue one) will appear more distant. The experiment may be varied by giving various sizes and shapes to the coloured disks, but with the same result, especially if the disks be looked at with one eye only. Orange and green disks may answer the purpose, but not so well as disks of red and blue, as these colours are most widely separated on the spectrum $†$.

* In 1835 Brewster wrote: "I consider the non-achromatism of the eye as a fact as well established as any other fact in natural philosophy," Phil. Mag. 1835, p. 161.

$\dagger$ Brewster called attention to similar phenomena produced by coloured outlines in 'Brit.-Assoc. Report,' 1848, p. 48, " On the Vision of Distance as given by Colour." He there gives a rather labuured explanation, conparing the vision of different distances for the differently coloured lines to the phenomena of the lenticular stereoscope. He adds that "the difference of distance of the coloured lines or spaces may be appreciated even with one eye." Compare also IIelnuloltz, Physiologisshe Optik, p. (445.

Compare also an experiment with a disk of black paper on a backgrouud of pale ultramarine, by Prof. O. N. Rood, 'Silliman's Journ.' xxxi. 1861, pp. 343,344 .

There is little doubt that Wheatstone's phenomenon of the "Fluttering Hearts" (see 'Brit.-Assoc. Rep.' 1844) is due to the attempt of the eyc to focus for adjacent spaces of colours of unequal refrangibility. which 
23. On this account I am disposed to think that retinal magnitude has little to do with the appreciation of distance, except in the case of objects whose magnitude and tint are familiar to us ; and I shall show other reasons for the opinion. Hence I regard as very imperfectly true the statement of the late Sir Charles Wheatstone, in the Bakerian Lecture for 1852 *, that "Convergence of the optic axes therefore suggests fixed distance to the mind; variation of retinal magnitude suggests change of distance." I think that the muscular sensation of convergence of the eyeballs serves rather to give an idea of rate of change of distance $\dagger$; and I have just indicated the cases to which variation of retinal magnitude are limited.

24. In Wheatstone's classical research of 1838 it was demonstrated how great is the capacity of the brain to combine together two slightly differing retinal images. This faculty once admitted, however explained, renders it unnecessary here to discuss how far the inexactness of focus for any particular tint is concerned in binocular vision. In the present paper it must suffice to treat of the non-achromatism of the eye in its relation chiefly to monocular vision. And, for reasons stated in $\S 19$, we shall omit further reference to linear perspective, since its relation to our perception of distance is not based upon apparent colour, or upon the formation of an exact retinal focus.

\section{Experimental Results, and Deductions.}

25. Fraunhofer $\ddagger$ is generally credited with the first careful attempt to measure the chromatic aberration of the eye. His method of experiment consisted in placing a wire in the principal focus of a telescope, in which the focus was then obtained by the adjustment of the lenses of an achromatic eyepiece. The theoretical adjustment to distinct vision for the

could not, therefore, be in distinct focus at one time. The same peculiar fluttering is observed when white objects on a dark ground are observed through a purple solution of permanganate of potash.

- Phil. Trans. 1852, p. 4.

+ See a curious little footnote by Mr. A. J. Ellis at p. 97 of his edition of Helmholtz's 'Sensations of Tone.'

†. See Gilbert s Annalen, lvi. 1814, p. 304; Helmholtz, Physiol. Optik; p. 128 ; Brewster, 'Edin. Phil. Journ,' xix. p. 25. 
various coloured rays was then calculated and compared with the actual adjustment; and the difference between theory and observation was assumed to measure the aberration of the eye. The method is at best only indirect; and there appears to be some doubt whether in the critical experiments an achromatic object-glass as well as an achromatic eyepiece was employed. Nevertheless, under the conditions of the experiment, the measurements were very accurately made, and are therefore invaluable.

26. I have found the most satisfactory exhibition of chromatic aberration to be manifested when a solution of permanganate of potash was employed, in a narrow glass trough, as a medium to intercept the middle rays of the spectrum. I have also tried solutions of iodine, and cobalt glasses of various depths of tint; but none are so satisfactory. For some experiments I have employed a piece of thin cobalt glass in conjunction with a solution of permanganate. By this means I have obtained media transmitting red and blueviolet rays freely, but totally opaque to all rays between $D$ and $\mathrm{E}$, and nearly opaque to rays from $\mathrm{C}$ to $\mathrm{D}$ in the orange, and from $\mathrm{E}$ to $\mathrm{F}$ in the green and blue.

27. A silvered bead placed in the sunlight, and viewed through such a medium can by no possibility be seen with accurate focus for all rays. It appears either as a near red point of light surrounded by a blue haze, or as a distant blue light surrounded by a red haze *. The point of light seems to change distance from far to near, or from near to far, with the effort of the eye. This effect, however, is only to be well observed when the intensities of the red and blue rays are about equal; for if either predominate, the eye will focus for the brighter unless special care is taken to adjust and keep. the required focus.

28. The researches of Jurin, Schreiner, Müller, Powell, and Trouessart have tended in favour of the view that the eye is achromatic at least for objects at the centre of the field of vision, when distinctly in focus. The fundamental illustration of this view is as follows:-Takeany visible white object-say

* This effect may be shown objectively to an entire audience by casting beams of light from a lamp through such a purple medium upon a silvered ball. 
a square inch of white paper upon a black background. If the eye be focused for a distance beyond the white surface, it will appear blurred at the edges, being tinged outwardly with orange-yellow, inwardly with blue. But if the eye be focused for a point nearer than the white surface, it will appear tinged outwardly with blue, in wardly with orange-yellow, while at exact focus these bordering tints disappear. To explain this seeming achromatism of the eye it was argued by Wollaston, Young, Muiller, and Matthiesen that the rays passed almost without refraction along the axis of the eyce, and therefore suffired no dispersion *. On the other hand, in my own experiments with permanganate-of-potash solution to intereept the yellow and green rays, I have never been able to obtain a luminous surface small enough to be free from aberration at the edges-which proves that the focus is really not exact. The rays of the middle of the spectrum, being more intense, mask the effects of the feebler rays; and the eyc focuses for the more intense light, as I have mentioned in $\S 27$.

29. If, on the contrary, a weak solution of picrate of potash be employed as a screen to cut off the extreme red and violet rays, it is very difficult with any focus to observe the tinted margins.

30. Moreover the apparent achromatisn of the experiment above mentioned is only found with pencils nearly coincident with the axis of the eye. Oblique pencils always give tinted margins in white light. This may be rerified by looking at a set of concentric circles in white and black. If the eye be steadily focused for the white centre, no colours are seen at its margin, but may be observed at the inner and outer edges of the other circles. I have always found it possible to get yellow exterior margins to a white object at any distance; that is, I have always found it possible to focus for a further distance; but not vice versâ.

31. If a square of white paper be carefully tinted around the edge with blue, and laid on a black ground, it appears

* Baden Powell contended that the refraction and dispersion of the vitreous lrumour being in the opposite sense to that of the crystalline lens, aqueous humour, and cornea, compensated the dispersion for axial pencils. 
further off than a perfectly white square of the same size beside it, since the blue margin helps to correct the yellow rays that surround the image at a longer focus. In short, a blue-edged square appears like a white square looked at with too near a focus. The opposite effect is observed with a margin tinted yellow.

32. I adjusted a lenticular stereoscope to easy focus, then placed in it a sheet of white paper having two equal circles 1 centim. in diameter, described upon it at the appropriate distance-one red, the other blue. The joint effect of these two gave, apparently, a combination of a red circle with a slightly larger one of blue. On drawing a slightly smaller circle of blue, with the circle of red as before, they appeared to give exact coincidence.

33. Knowing the power of the eye to reconcile retinal images in magnitude (see $\$ 16$ ), I placed in the stereoscope a penny coloured blue and a halfpenny coloured red. They refused to be seen as one object. On replacing them by a red penny and a blue halfpenny, coincidence was, with some difficulty, obtained.

34. My next experiments attempted the direct estimation of the distance of objects by the focus of the eye apart from other means of estimation. A conical tube of metal, blackened interiorly, was taken, through which only a limited field of view was possible, the eye being placed at the smaller aperture. In front of this tube a screen of blackened cardboard was placed, capable of adjustment to any distance from 10 centims. to 350 centims. from the tube, and covered the entire field of view. Coloured glasses could be introduced in front of the tube. The objects displayed upon the cardboard screen in the centre of the field of vision were irregularly shaped pieces of white paper. These were cut by an assistant and adjustedtheir distance and size being unknown to the observer, and varied between each experiment. Hence, under the conditions given, the eye could form no judgment of distance based upon apparent magnitude, upon parallax, or upon apparent form or shadow; the estimation depended solely upon the muscular sensation of adjustment to focus. After a little practice this kecame moderately casy with white light, and the estimations were fairly within limits. With a screen 
of yellow glass interposed no difference was experienced. When a ruby-red glass was employed, however, the estimations were generally, though not invariably, too small, and with a cobalt-blue glass generally (though not invariably) too great. The following is the mean of six experiments taken at random from a number made with a red glass, and of another six from those made with a blue glass.

Red.

Blue.

Mean real distance ...... 208 centims.

Mean estimated distance $163 \cdot 1$,

178 centims.

199 ,

35. When a solution of permanganate was employed in conjunction with a blue glass, giving equal intensities of red and blue rays, the attempt to estimate the distance of the white pieces of paper became perplexing in the extreme. The object seemed to be moving backwards and forwards as the focus changed from red to blue and blue to red.

36. After about forty experiments with blue and red glasses, the errors became less. Evidently the effect of practice was to sophisticate the result, the mind knowing the probable result. beforehand, and making allowance involuntarily. The forty experiments were not all made at one time, because the eye becomes fatigued when experimentation is prolonged; and as in my own case with fatigue partial astigmatism sets in, I was careful to test my vision from time to time, to avoid error from this source*.

37. Every book on Art will tell us that blue is a " retiring" colour, while red is an "advancing colour." For long I had been convinced that these alleged characteristics were something more than associations with blue sky and red rocks; and the universality and tenacity with which artists pronounce

* Since the experimental results recounted in this paper have been chiefly obtained with my own eyes, it may be well to state the following particulars:-For objects in centre of field of vision there is no trace of colour-blindness; neither eye is astigmatic when not fatigued; their range of accommodation is from 11 centims. to infinity, and with good definition at all ranges; the blind-spots are distinct, the fover centrales well formed and sensitive; there is no difficulty in perceiving Purlinje's figures and Haidinger's brushes and lavender streaks. Let me add, as a test of definition, that on a clear night I can easily see twelve stars of the Pleiades. 
this opinion is surely indicative of a reality somewhere. The opinion of Goethe on this point sums up the whole matter. I quote from Sir Chas. Eastlake's edition of the 'Farbenlehre ${ }^{\prime *}$.

"As the upper sky and distant mountains appear blue, so a blue surface seems to retire from us."

"Rooms which are hung with pure blue appear in some degree larger, but at the same time empty and cold."

38. So the blueness of the lower misty air may, as Alpine travellers know, cause the base of a mountain actually to appear more distant than its summit. Mr. Ruskin has noted this in his 'Modern Painters' + ; and the fact bas been familiar to artists for centuries, having been described by Leonardo Da Vinci in his Trattato della Pittura, and is alluded to by Goethe.

39. The sun and moon when red at setting or rising, and the moon when red in total eclipse, look nearer-or, some people say, larger-than at other times.

40. For the sake of giving prominence or nearness to the foreground of a picture, painters will contrive to introduce a scarlet flower, or a patch of red rock, or a figure dressed in red-a practice sanctioned and well known in Art, but quite inexplicable except on the train of reasoning we have been attempting to follow.

41. The immense importance of these facts of physical and physiological optics has long been recognized in Art. The study of aerial perspective has been carried long since to a pitch that renders it worthy to be put upon a basis more than merely empirical. There can be little doubt that other empirical laws may be similarly accounted for. Visitors to picture-galleries may be constantly observed scanning a landscape through a rolled up catalogue, or under the arched fingers of the hand. Is there not an explanation, too, for this? The square frame of gold asserts the flatness of the surface, and prevents the mind from realizing that which the eye perceives - that all the rays of light do not focus at once upon the retina, but that each tint retires or advances to its

* Goethe's 'Theory of Colours,' translated by Sir C. L. Eastlake, R.A., F.R.S. (London: 1840), p. 311, par. 780 and 783.

+ Vol. i. p. 182. 
own appropriate distance. Landscapes without figures or architecture, especially, are inproved by this method of treatment; and the reason is plain: in them the eye judges the scene by colour, not by retinal magnitudes or binocularly compound sensations.

42. I have endeavoured to apply the method of experimentation in another direction. Take any picture in which there are well-expressed foregrounds and distances, and look at it through the coloured media employed in the earlier experiments. If the facts of distance are expressed only indirectly, by suggestions of magnitude, by linear perspective of vanishing lines and the like, the interposition of a red or a blue glass will produce little effect, except that the latter may deaden the .intensity of the shadows. If, however, the expression of distance in the picture is accomplished chiefly by aerial perspective (that is to say, by colour), a red glass will almost destroy the intelligibility of the picture, while a blue glass will draw out the distances in a marked manner.

43. Reflecting how useful is the purpose subserved thus by the non-achromatism of the eye, I consider it probable that if the eye were so constructed as to be originally achromatic, having usually blue distances and red-brown foregrounds to look at, it would, by an inevitable process of natural selection, develop into a non-achromatic instrument.

\section{Summary and Recapitulation.}

44. I would conclude therefore :-

(a) That the muscular sensation of the adjustment to focus of the lenses of the eye affords a possible means of estimating distances.

( $\beta$ ) That when binocular methods, and those depending on association of visible form or magnitude fail, the eye falls back on colour as a means of estimating distance.

( $y$ ) That estimates of distance founded on apparent magnitude are liable to be rendered fallacious by the colour of the object.

( $\delta$ ) That, conversely, estimates of distance founded on colour are liable to be confused by apparent magnitude.

(є) That colour may in some cases outweigh, as a criterion, the evidence of binocular vision. 
Proc. Phys. Soc. Vol . 2.Pl.III.
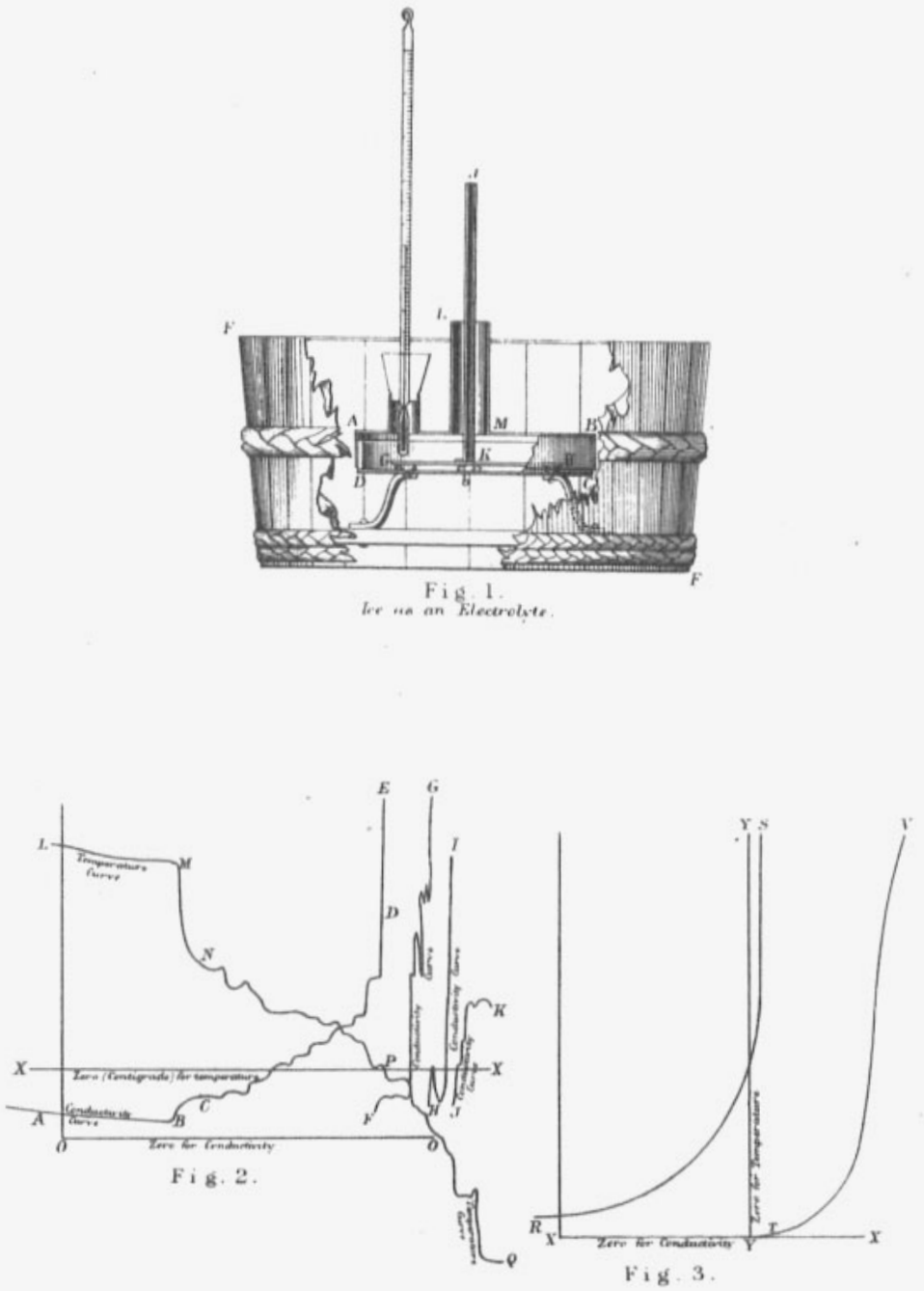


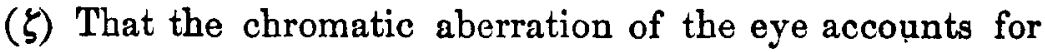
the universal opinion of painters as to the "retiring" character of blue, and the "advancing" character of red tints.

$(\eta)$ That Aerial Perspective in Art is a true expression of a physical fact in the perception of distance.

University College, Bristol, May $8,187 \%$.

XVI. Ice as an Electrolyte. By W. E. Ayrton and JoHN Perry, Professors in the Imperial College of Engineering, Tokio, Japan.

\section{[Plate III.]}

For the purpose of measuring the resistance of ice at various temperatures, its power to act as an electrolyte, and its specific inductive capacity, the following piece of apparatus was constructed. A BCD (Plate III. fig. 1) is a copper box 17.4 centims. in diameter, rigidly fixed by means of three legs in the inside of a wooden tub F F. Resting on the bottom of the copper box, but separated from it by three small pieces of glass (a) (b) (c), is a copper disk, $\mathrm{GH}, 13$ centims. in diameter, to which is fastened a copper strip $\mathrm{JK}$. The box is closed by a very tightly fitting cover furnished with two openings--one, $\mathrm{L} \mathrm{M}$, to allow $\mathrm{J} \mathrm{K}$ to pass through without touching the cover, the other for the admission of a thermomeier. Distilled water having been introduced into the copper box to a sufficient depth to cover the disk $\mathrm{GH}$, the temperature could be lowered by the introduction of a freezing-mixture of snow and salt inside the tub above and below the copper box, great care being taken that none of the mixture fell into the box through the tube $\mathrm{L} M$, which was necessarily left open.

I. Preliminary experiments.--The current from one Meidinger cell was passed through the ice and a reflecting-galvanometer, the space between the copper plates being 2 millims. Keeping the temperature nearly constant (it varying between $-18^{\circ .2}$ and $-17^{\circ} .2$ C.), we found that in one hour the galvanometerreadings increased from 46.8 to $168 \cdot 8$, corresponding to a diminution in resistance per cubic centimetre of the ice from 